\title{
An fMRI Study of the Effect of Amphetamine on Brain Activity
}

\author{
Stephen J. Uftring, Ph.D., Stephen R. Wachtel, Ph.D., David Chu, Ph.D., Cyrus McCandless, B.S., \\ David N. Levin, M.D., Ph.D., and Harriet de Wit, Ph.D.
}

Functional magnetic resonance imaging was used to evaluate the effects of oral d-amphetamine on brain activation elicited by auditory and simple motor tasks in ten normal right-handed subjects. We measured the percent signal change and number of voxels activated by a tone discrimination task and a right hand finger-tapping task after $20 \mathrm{mg}$ of d-amphetamine and after placebo. Compared to placebo, amphetamine significantly increased the number of activated voxels in the left and right primary auditory cortices during the tone discrimination task and increased the number of activated voxels in the ipsilateral primary sensorimotor cortex and right middle frontal area during the motor task. Although highly specific vascular effects of drug cannot be ruled out as an explanation, these results could also mean that amphetamine increases the neuronal activity associated with each of these two tasks.

[Neuropsychopharmacology 25:925-935, 2001]

(C) 2001 American College of Neuropsychopharmacology.

Published by Elsevier Science Inc.
KEY WORDS: Amphetamine; fMRI; Brain mapping; Motor task; Tone decision task

Recent advances in brain imaging technology, including the technique of functional magnetic resonance imaging (fMRI), have provided researchers the opportunity to study regional brain activity while subjects are in various emotional states or while they are performing cognitive and motor tasks (Cohen and Bookheimer 1994). One of the applications of fMRI has been to study the specific neural actions of drugs of abuse (Kleinschmidt et al. 1999). Typically, these studies are designed to investigate regional changes in neural activa-

From the Department of Radiology (SJU, DC, DNL) and Department of Psychiatry (SRW, CM, HdW), The University of Chicago, Chicago, IL.

Dr. David Chu's present address: Fonar Corporation, Melville, NY.

Address correspondence to: Stephen J. Uftring, Ph.D., The University of Chicago, Department of Radiology, MC 2026, 5841 S. Maryland Ave., Chicago, IL 60637.

Received 16 October 2000; revised 16 February 2001; accepted 20 June 2001.

Online publication: 6/27/01 at www.acnp.org/citations/ Npp062701141. tion in the presence or absence of a certain drug, either alone or during a particular activational state. The activational state may consist of performance of a specific cognitive or motor task, or simple exposure to a specific visual or auditory stimulus. Several previous studies have explored the effects of stimulant drugs, such as cocaine or amphetamine, on brain activity during auditory or visual stimulation (Howard et al. 1996; Gollub et al. 1998), during presentation of a drug-related visual cue (a stimulus used to induce craving (Maas et al. 1998)), or during performance of the Wisconsin Card Sorting Task (a measure of executive function (Daniel et al. 1991)). The results of these studies indicate that stimulant drugs may increase neural activity in specific regions of the brain when subjects are engaged in particular tasks, and the particular brain regions activated correspond well with known functional neuroanatomy. Thus, the fMRI technique makes it possible to study the mechanisms whereby psychoactive drugs alter behavior and may advance our understanding of brain function and organization.

In the present study, we examined the effects of a moderate dose of $d$-amphetamine $(20 \mathrm{mg}$ ) on brain ac- 
tivity while subjects performed two tasks, an auditory vigilance task and a simple motor task. Amphetamine has well-documented effects on psychomotor and cognitive function (Koelega 1993), and there is some evidence that its effects on vigilance are more pronounced than its effects on motor performance. Two previous studies have shown that low doses of amphetamine (5 to $10 \mathrm{mg}$ ) improve performance of an auditory vigilance task, while leaving a simple motor task (finger tapping) unaffected (Bye et al. 1973; Hamilton et al. 1983). This study of brain activity after administration of amphetamine and during performance of two tasks allowed us to examine the relationship between changes in regional brain activity and changes in behavior induced by the drug. Here we report the effects of $20 \mathrm{mg}$ of amphetamine, administered to 10 healthy volunteers under double-blind conditions, on neural activity during performance of an auditory vigilance task and a finger-tapping task.

\section{METHODS}

\section{Subjects}

The subjects were ten healthy males who were determined to be right-hand dominant according to the Edinburgh Handedness Inventory test (Olfield 1971). The subject demographics and drug use are summarized in Table 1. Prior to participation, all subjects underwent a structured clinical interview and physical examination that included an electrocardiogram.

Exclusion criteria were: any current medical condition requiring medication; any current or past medical condition considered to be a contraindication for the

Table 1. Subject Demographic And Drug Use Summary $(n=10)$

\begin{tabular}{lc}
\hline Age (years) & \\
Range & $18-34$ \\
Mean \pm SD & $25.7 \pm 5.1$ \\
Weight (lbs: mean \pm SD) & $167.6 \pm 25.1$ \\
Race/ethnicity & \\
Caucasian & 9 \\
African-American & 1 \\
Education (n) & \\
Partial college & 4 \\
College degree & 6 \\
Drug use & \\
Alcohol (mean \pm SD; drinks/week) & $2.8 \pm 2.1$ \\
Caffeine (mean \pm SD; drinks/week) & $2.5 \pm 4.8$ \\
Cigarettes $(n ;>2.5$ cigarettes/day) & 2 \\
Stimulants $(n ;$ ever used) & 1 \\
Tranquilizers $(n ;$ ever used) & 1 \\
Hallucinogens $(n ;$ ever used) & 3 \\
Opiates $(n ;$ ever used) & 3 \\
Marijuana $(n ;$ ever used) & 8 \\
Inhalants $(n ;$ ever used) & 1 \\
\hline
\end{tabular}

study (e.g., hypertension); any current Axis I psychiatric disorder (American Psychiatric Association 1994); a history of psychosis; a history of drug or alcohol dependence, including any treatment for a substance use disorder; a history of legal, personal, or employment problems related to drug use; less than a high school education; lack of fluency in English; night shift work; and total abstention from all drugs including alcohol.

\section{Procedure}

The study was approved by the Institutional Review Board at The University of Chicago in accordance with the Code of Federal Regulations (Title 45, Part 46) "Protection of Human Subjects" as adopted by the National Institutes of Health and the Office for Protection from Research Risks, and was conducted ethically in accordance with the Helsinki Declaration of 1964 (revised 1989) and the National Advisory Council on Drug Abuse Recommended Guidelines for the Administration of Drugs to Human Subjects. Subjects provided informed consent at an orientation session at which the rules and conditions of participation were carefully explained. For blinding purposes, subjects were instructed that they might receive any of a number of drugs (e.g., stimulant, sedative, antihistamine, and placebo). They were instructed not to take any drugs other than their usual amounts of caffeine and nicotine for 24 hours before and 24 hours following each session. Before each session, urine was collected and tested for amphetamine, cocaine, PCP, opiate, and marijuana use with Ontrak TesTstik ${ }^{\mathrm{TM}}$ test kits (Roche Diagnostic Systems, Inc., Somerville, NJ), and breath alcohol level was assessed using an Alco-Sensor III hand-held Breathalyzer (Intoximeters, Inc., St. Louis, MO). None of these were positive.

The study utilized a two-session, placebo-controlled, crossover design. Each subject received d-amphetamine $(20 \mathrm{mg})$ or placebo in randomized order under double blind conditions during two laboratory sessions, one week apart. During each session, prior to ingestion of the capsule, baseline physiological and subjective effect data were collected (see Dependent Measures). Two hours after ingesting the capsule, subjects underwent an fMRI imaging procedure during which they performed simple auditory and motor tasks (see below). Physiological and subjective effects measures were collected before taking the capsule, and at 60 and $120 \mathrm{~min}$ after taking the capsule. These measures were also collected just after the fMRI scans, approximately $180 \mathrm{~min}$ after taking the capsule, and again at the end of the session, $4 \mathrm{hrs}$ after the capsule. After completing the study, subjects attended a debriefing session and received payment. 


\section{Drugs}

d-Amphetamine (5 mg; Dexedrine $\left.{ }^{\circledR}\right)$ tablets were placed in opaque, colored gelatin capsules (size 00) with dextrose filler. Placebo capsules contained only dextrose. The moderate dose of $d$-amphetamine (20 mg) was chosen because it reliably produces increases on a variety of subjective psychostimulant measures described below (Martin et al. 1971; Foltin and Fischman 1991a,b; Brauer and de Wit 1995, 1997; de Wit et al. 1997; Wachtel and de Wit 1999).

\section{fMRI Protocol}

All imaging was performed with a $1.5 \mathrm{~T}$ clinical system (Signa; GE Medical Systems, Milwaukee, WI). A vacuum bag (MED-TEC, Inc., Orange City, IA) was used to minimize the head motion of the subjects. A volumetric T1-weighted gradient echo scan (60 contiguous sagittal slices, $2.8 \mathrm{~mm}$ slice thickness, in-plane resolution $1 \mathrm{~mm} \times$ $1.9 \mathrm{~mm}$ ) was used to locate slice positions for the fMRI scans and to provide anatomical images on which functional activation maps could be superimposed. The fMRI scans were performed with a single-shot T2* EPI pulse sequence having the following parameters: TR/ TE $5000 \mathrm{~ms} / 60 \mathrm{~ms}, 6 \mathrm{~mm}$ slice thickness, $1 \mathrm{~mm}$ interslice spacing, $24 \mathrm{~cm}$ FOV , $3.75 \mathrm{~mm} \times 3.75 \mathrm{~mm}$ in-plane resolution. The entire brain was imaged with 20 sagittal slices during the tone decision (TD) task and with 20 axial slices during the finger-tapping (FT) task.

The fMRI images were acquired while the subject performed either a TD task or a FT task (see below) using a boxcar paradigm that consisted of five 50-second task periods alternating with five 50 -second control periods. Data acquisition during the task period began 10 sec after the subject had started the task to allow activated regions to reach their peak blood flow. Task and control scans were separated from each other by nonscanning periods of 30 seconds to allow time for the hemodynamic changes associated with the task performance and scanner noise to return to baseline levels. This insured that there was no carry over of activation between conditions. The brain was scanned every 5 seconds giving a total of 80 time points for each paradigm.

The TD paradigm followed the procedure of Binder et al. (1997). During the 50-second task period, the subject was presented with multiple short series of randomly distributed high $(750 \mathrm{~Hz})$ and low $(500 \mathrm{~Hz})$ tones. The tones were generated by a Macintosh computer and were delivered through headphones containing MRI-compatible electromechanical transducers (Resonance Technologies, Inc., Northridge, CA). Figure 1 shows a schematic of a typical part of the tone presentation protocol. Each series consisted of 3-7 tones with $150 \mathrm{msec}$ tone duration and $250 \mathrm{msec}$ between tones. There was 1 second of silence between series, during which the subject was instructed to press a button on a MRI-compatible mouse if he heard two and only two high tones, in any order, in the preceding series. During the control periods for this task, the subject relaxed in the presence of no sound except scanner noise. During the task, both reaction time (time between the end of the stimulus and the response) and response accuracy (number of correct responses) were recorded. Because the first two subjects participated before appropriate equipment was available, these performance measures were only collected for eight of the 10 subjects.

For the FT task, the subjects were asked to continually touch their finger to their thumb in a series of sequential oppositions to the thumb, in a self-paced fashion, at the fastest consistent pace that the subject could manage (Cao et al. 1993; Rao et al. 1993, 1996). Only the subject's dominant right hand was tested. No performance data was collected. During the control period, the subject was told to relax; no task was performed.

\section{Image Analysis}

Functional MRI data were analyzed by a two-step procedure. First, a "group-average" activation map was created from the data acquired from the 10 subjects while they performed a given task (TD or FT) in the presence or absence of drug. This "group-average" activation map was simply used to identify regions of interest (ROIs) within which significant activation occurred during each type of task. Second, individual activation maps were created from the data acquired from each subject while he performed a given task, and the volume of activation and the average percent signal change in each ROI was computed for each individual.

The images from each experiment were first registered with a motion correction algorithm (AIR 3.0) (Woods et al. 1992), and the signal time series in each voxel was linearly detrended with the AFNI software package (Cox 1996). For each subject in each condition (TD and FT, amphetamine and placebo), an individual correlation map was generated by cross correlating the time course of the fMRI signal in each pixel with a

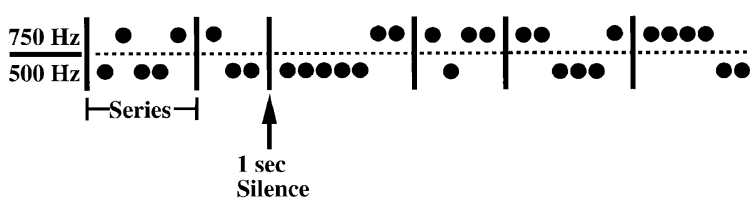

Figure 1. Sample tone pattern for the TD paradigm. Dots indicate a single $150 \mathrm{msec}$ tone. Tones were separated by 250 msec, and each series of tones were separated by a $1 \mathrm{sec}$ response interval. In this example, the first and third series should be followed by a button push (i.e., positive response). 
square wave reference waveform that followed the periodicity of the boxcar paradigm.

Because a square wave reference waveform was used, there was a one-to-one correspondence between the cross correlation coefficient (cc) and the t-statistic (Ardekani and Kanno 1998). These individual correlation maps and the individual T1-weighted structural images were transformed into Talairach space (Talairach and Tournoux 1998) with the aid of anatomical landmarks that were located on the structural scans. The Talairach-transformed correlation maps voxels were then linearly resampled to $4 \times 4 \times 4 \mathrm{~mm}$. These correlation maps were combined for the ten subjects who performed the same task in the presence of amphetamine or placebo. This was done via the Fisher transformation (Anderson 1984) where the cc is converted to a $z$-statistic that is distributed almost normally. These values were then averaged across individuals, and the reverse-transformation was applied to give the "group-averaged" correlation coefficient map. The "group-averaged" cc map was thresholded to identify "active" voxels, i.e., those voxels that have a significant change in MR signal between the task and control periods. "Active" voxels were those that had a cc $\geq 0.30$ ( $p<1 \times 10^{-10}$, uncorrected for multiple comparisons). These "active" voxels were also required to be part of a cluster of five or more contiguous voxels $\left(320 \mathrm{~mm}^{3}\right)$, each of which had a "group-average" $c c \geq 0.30$. This was done in order to discriminate against foci of false positive activity, which tend to be single voxels or small clusters of voxels (Forman et al. 1995). Voxels that survived the threshold and cluster requirements were then color-coded to indicate foci of activation in each condition (amphetamine or placebo). The images of these voxels comprised the so-called "group-average" activation maps.

The Talairach-transformed T1-weighted images were also averaged across subjects to produce images of "average brain anatomy". The color-coded "groupaverage" activation maps were overlaid on the "average brain" images so that the anatomical locations of activated foci could be clearly identified. Next, the ROI for each focus of activation was defined in the "groupaverage" activation maps. Each ROI was defined to be smallest axially oriented rectangular solid that included all of the voxels in a given focus of activation in the amphetamine or placebo condition.

Finally, the volume of activation and the percent signal change in each ROI was computed for each individual based on his individual activation map for each condition. To create an individual activation map, the correlation map (in Talairach space, $4 \times 4 \times 4 \mathrm{~mm}$ ) of each individual was thresholded with a correlation coefficient $=0.60$. This corresponds to a single-voxel t-test with significance of $p<5.1 \times 10^{-9}$. Monte-Carlo simulations (the AlphaSim program of the AFNI suite) (Cox
1996) estimated the false positive probability, $\alpha$, to be less than 0.0001 . This calculation accounted for the effect of comparisons across multiple voxels and included an estimate of the spatial correlation between voxels (intrinsic gaussian blur within the image). For each individual the number of voxels that survived the threshold criteria was then tallied in each ROI and the average percent signal change of these surviving voxels was calculated.

\section{Physiological and Subjective Effect Measures}

Blood pressure and heart rate were assessed using a Digital Blood Pressure Monitor HEM-706 (Omron Healthcare, Inc., Vernon Hills, IL). Subjective drug effects were determined using a 49-item version of the Addiction Research Center Inventory (ARCI) (Martin et al. 1971) that contains true or false statements sensitive to the effects of several drug classes. This version of the ARCI has five empirically derived scales: the Amphetamine (A), Benzedrine Group (BG), the Morphine-Benzedrine Group (MBG), the Pentobarbital-Chlorpromazine-Alcohol Group (PCAG), and the Lysergide (LSD). Subjects also reported subjective effects on five of visual analog scales: "stimulated", "sedated", "hungry", "anxious", and "nauseous" (VAS) (Folstein and Luria 1973).

For each item, subjects were required to rate on 100$\mathrm{mm}$ lines the extent to which they feel each adjective, from "not at all" on the left end of the scale to "extremely" on the right end of the scale. Subjects completed a Drug Effects Questionnaire that contained four $100 \mathrm{~mm}$ visual analog scales that the subjects used to mark their response the following questions: 1) Do you feel any drug effects, rated from "none at all' to "a lot"; 2) Do you like the effects you are feeling now, rated from "dislike" to "like very much"; 3) Are you high (as in drug high), rated from "not at all" to "very"; and 4) Would you like more of what you consumed, right now, rated from "not at all" to "very much", used to assess how much the subjects "want" the drug. These questionnaires are sensitive to the dose-related effects of a variety of psychoactive drugs (Foltin and Fischman 1991a,b; Fischman and Foltin 1991).

\section{Data Analysis}

Data on activation volumes and percent signal change for each task (TD and FT) and for each ROI were analyzed using a paired Wilcoxon signed rank test to test if amphetamine significantly increased the number of activated voxels, or significantly increased the percent signal change when compared to the placebo condition. Reaction time and performance data from the TD task were compared using paired t-tests. Physiological and subjective effect data were analyzed using two-factor repeated measures ANOVA with DRUG (two levels; am- 
phetamine and placebo) and HOUR (time within session) as the two factors. Post-hoc comparisons limited to drug and time-dependent effects were conducted with the Fisher least significant difference test. The significance level for all statistical tests was set at $p<0.05$.

\section{RESULTS}

\section{Physiological and Subjective Effects}

The effects of amphetamine on vital signs and subjective state were typical of those reported in several other recent studies from our laboratory (Brauer and de Wit 1995, 1997; de Wit et al. 1997; Wachtel and de Wit 1999). Amphetamine increased heart rate (DRUG $\times$ HOUR interaction; $\mathrm{F}(4,36)=13.45, p<.0001)$, systolic blood pressure (DRUG $\times$ HOUR interaction; $\mathrm{F}(4,36)=2.67, p<$ .05 ), and diastolic pressure (DRUG $\times$ HOUR interaction; $\mathrm{F}(4,36)=9.94, p<.01)$. Amphetamine also increased subjective amphetamine-like effects as measured by the ARCI amphetamine scale (Figure 2; main effect of DRUG; $\mathrm{F}(1,9)=6.70, p<.05)$ and euphoria as measured by the ARCI MBG scale (DRUG $\times$ HOUR interaction; $\mathrm{F}(4,36)=3.11, p<.05)$.

On VAS scales, subjects' self-reported amphetamineinduced increases in feelings of "stimulated" (Figure 2; DRUG $\times$ HOUR interaction; $\mathrm{F}(4,36)=3.67, p<.05)$, and significant effects (DRUG $\times$ HOUR interactions) on each question of the Drug Effects Questionnaire. This included increases in feeling of a drug effect $[\mathrm{F}(4,36)=$ $5.15, p<.01]$, feeling a drug high $[\mathrm{F}(4,36)=5.68, p<$ $0.01]$, liking the drug effect $[\mathrm{F}(4,36)=4.97, p<.01]$, and wanting more drug $[\mathrm{F}(4,36)=5.86, p<.01]$.

\section{Motion during fMRI}

Gross motion of the subject's head during an fMRI scan can cause an apparent increase in the number of acti-

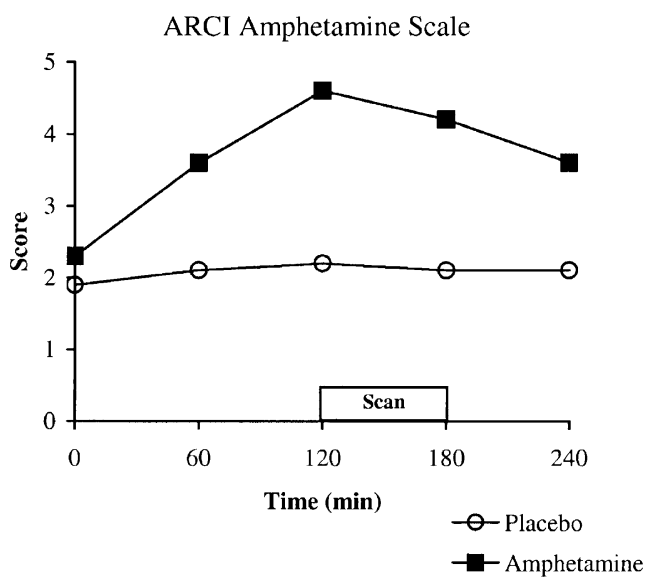

vated voxels. To determine whether amphetamine caused more motion than placebo, estimated motion parameters were obtained from the motion correction algorithm. Table 2 shows the values for each task (TD and FT) for drug and placebo. The rotations are defined as follows: Pitch is a rotation about the right-left axis of the subject; Roll is a rotation about the inferior-superior axis of the subject; and Yaw is a rotation about the anterior-posterior axis of the subject. The translations are defined as: $X$-Trans is along the right-left axis of the subject; $Y$-Trans is along the inferior-superior axis of the subject; and Z-Trans is along the anterior-posterior axis of the subject. Amphetamine did not significantly change these motion parameters (paired Wilcoxon sign rank test). Although theoretically it is possible that even non-significant motion could influence the activation maps, this increase should be apparent in all active regions, whereas the increases observed here were specific to a few regions.

\section{Tone Decision}

Figure 3 shows the "group-average" activation maps for the TD task superimposed upon an "average" image of brain structure for both the amphetamine and placebo conditions. The areas of activation closely replicate those observed by Binder et al. (1997). The ROIs defined from these functional images corresponded to the following regions according to the Talairach atlas: left primary auditory cortex (LPAC), right primary auditory cortex (RPAC), supplemental (right and left) motor cortex (SMA), thalamus (THAL), left cerebellum (LCB), right superior parietal area (RSP), right middle frontal area (RMF), left middle frontal area (LMF), left superior temporal area (LST), right inferior frontal cortex (RIF), left inferior frontal cortex (LIF), left superior temporal gyrus (LSTG), and left inferior parietal area (LIP). Fig-

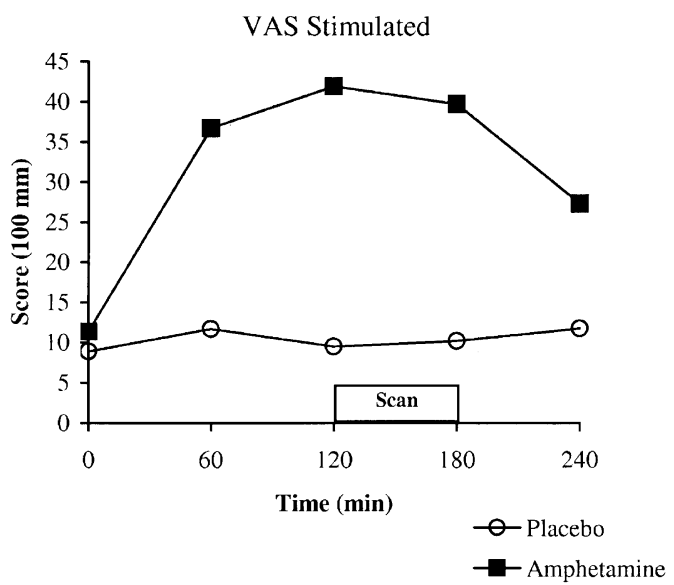

Figure 2. Effects of amphetamine on standardized measures of subjective drug effects. Data represent the mean \pm SEM. Amphetamine $(20 \mathrm{mg}$ ) was ingested orally at time $0 \mathrm{~min}$. 
Table 2. Average Motion Parameters ( \pm SD) for The Three Rotations (Pitch, Roll, and Yaw) in Degrees (deg) And for The Three Translations (X, Y, and Z) in Millimeters (mm), for Each Task (TD And FT) And for Each Condition (Drug and Placebo)

\begin{tabular}{lcccr}
\hline & \multicolumn{2}{c}{ Tone Decision } & \multicolumn{2}{c}{ Finger Tapping } \\
\cline { 2 - 3 } & Amphetamine & Placebo & Amphetamine & Placebo \\
\hline Pitch (deg) & $0.75 \pm 0.41$ & $0.54 \pm 0.22$ & $0.36 \pm 0.23$ & $0.30 \pm 0.32$ \\
Roll (deg) & $0.08 \pm 0.01$ & $0.06 \pm 0.10$ & $0.69 \pm 0.31$ & $0.36 \pm 0.10$ \\
Yaw (deg) & $0.03 \pm 0.01$ & $0.04 \pm 0.04$ & $0.23 \pm 0.15$ & $0.19 \pm 0.12$ \\
X-Trans (mm) & $0.05 \pm 0.01$ & $0.03 \pm 0.05$ & $0.27 \pm 0.26$ & $0.36 \pm 0.13$ \\
Y-Trans (mm) & $0.71 \pm 0.25$ & $0.76 \pm 0.42$ & $0.60 \pm 0.21$ & $0.27 \pm 0.20$ \\
Z-Trans (mm) & $0.37 \pm 0.20$ & $0.31 \pm 0.11$ & & $0.53 \pm 0.36$ \\
\hline
\end{tabular}

The rotations are defined as follows: Pitch is a rotation about the right-left axis of the subject, Roll is a rotation about the inferior-superior axis of the subject, and Yaw is a rotation about the anterior-posterior axis of the subject. The translations are defined as: X-Trans is along the right-left axis of the subject, Y-Trans is along the inferior-superior axis of the subject, and Z-Trans is along the anterior-posterior axis of the subject. None of the differences between amphetamine and placebo were significant.

ure 4 shows the average number of activated voxels $(4 \times$ $4 \times 4 \mathrm{~mm}$ ) for each ROI based on each individual subject's activation map for the amphetamine and placebo sessions.

Compared to placebo, amphetamine produced significantly greater activation, i.e., more active voxels, in both the LPAC $(p<.01$, uncorrected for multiple ROI comparisons) and RPAC ( $p<.02$, uncorrected for multiple ROI comparisons) during the TD task. However, the number of voxels activated in all the other regions was not significantly affected by amphetamine. Table 3 shows the average percent signal change for each ROI. Amphetamine produced no significant affect in percent signal change in any of the activated regions. In addition, amphetamine did not significantly affect either response reaction time or the number of correct responses on the tone task (Figure 5).

\section{Finger Tapping}

Figure 6 shows the "group-average" activation maps for the FT task superimposed on images of "average" brain structure. The areas of activation are similar to

Table 3. Average Percent Change of fMRI Signal in Each ROI for TD Task ( \pm SEM)

\begin{tabular}{lcc}
\hline ROI & Amphetamine & Placebo \\
\hline LPAC & $2.7 \pm 0.2$ & $2.1 \pm 0.3$ \\
RPAC & $2.9 \pm 0.4$ & $2.2 \pm 0.4$ \\
SMA & $1.7 \pm 0.3$ & $2.0 \pm 0.4$ \\
THAL & $1.7 \pm 0.6$ & $1.5 \pm 0.5$ \\
LCB & $1.8 \pm 0.4$ & $1.8 \pm 0.5$ \\
RSP & $2.1 \pm 0.4$ & $2.3 \pm 0.4$ \\
RMF & $2.5 \pm 0.4$ & $2.4 \pm 0.3$ \\
LMF & $1.6 \pm 0.4$ & $1.3 \pm 0.4$ \\
LST & $1.1 \pm 0.5$ & $0.6 \pm 0.4$ \\
RIF & $2.4 \pm 0.8$ & $2.4 \pm 0.6$ \\
LIF & $1.4 \pm 0.6$ & $0.4 \pm 0.3$ \\
LSTG & $0.6 \pm 0.3$ & $0.6 \pm 0.3$ \\
LIP & $0.8 \pm 0.4$ & $0.7 \pm 0.4$ \\
\hline
\end{tabular}

those observed by other investigators in both fMRI (Cao et al. 1993; Rao et al. 1993; Schubert et al. 1998) and positron emission tomography (PET) (Fink et al. 1997) experiments. The ROIs defined from these images corresponded to the following regions of the Talairach atlas: contralateral (left) primary sensorimotor cortex (CPSM), ipsilateral (right) primary sensorimotor cortex (IPSM), supplemental (right and left) motor cortex (SMA), ipsilateral (right) cerebellum (ICB), contralateral (left) cerebellum (CCB), right middle frontal area (RMF), left middle frontal area (LMF), right inferior frontal cortex (RIF), left inferior frontal cortex (LIF), thalamus (THAL), and putamen (PUT).

Figure 7 shows the average number of activated voxels $(4 \times 4 \times 4 \mathrm{~mm})$ for each ROI from each subject's activation map for the amphetamine and placebo sessions. Amphetamine significantly increased the number of active voxels in both the IPSM $(p<.01$, uncorrected for multiple ROI comparisons) and RMF ( $p<.03$, uncorrected for multiple ROI comparisons). The number of voxels in all other regions activated by FT was not significantly affected by amphetamine. Table 4 shows the average percent signal change for each ROI. Amphetamine produced no significant affect in percent signal change in any of the activated regions.

\section{DISCUSSION}

The results of the present study demonstrate that fMRI provides a highly sensitive measure of psychostimulant effects on both auditory and motor related brain function in humans. Amphetamine produced significant region-specific changes in the volume of brain activity related to an auditory attention task, even though there was no apparent change in performance. Similarly, amphetamine produced region-specific changes in brain activation during a simple motor task even though previous reports suggest that this dose of amphetamine does not affect performance of simple motor tasks (Bye 


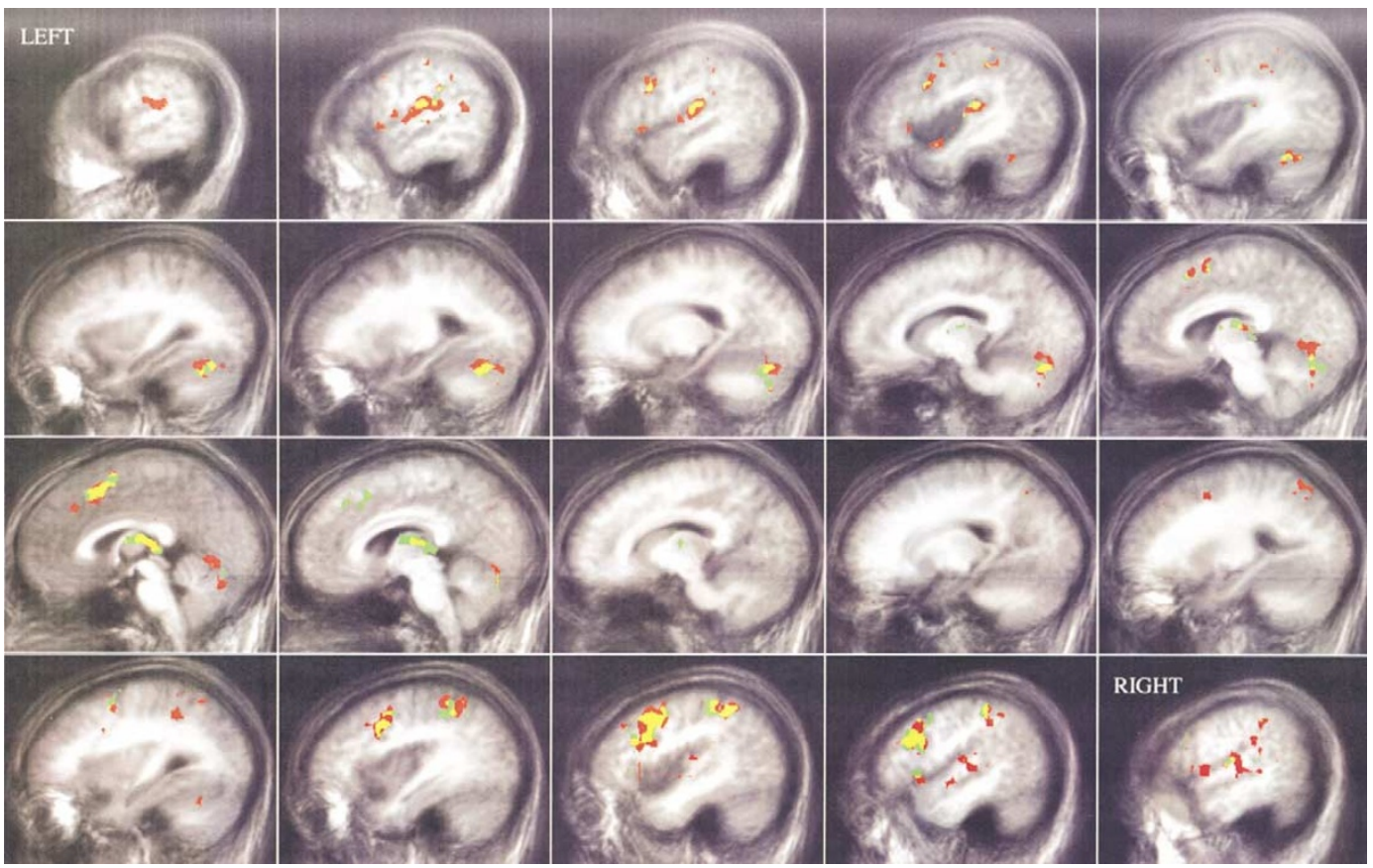

Figure 3. Map of brain activation for all subjects as a group ("group-average" activation map) during the tone decision task. Red areas indicate activation for amphetamine only. Green areas indicate activation during placebo only. Yellow areas indicate activation during both amphetamine and placebo conditions.

et al. 1973; Hamilton et al. 1983). The brain areas activated by the tasks, and those increased by amphetamine, correspond with areas known to be involved in auditory or motor performance. Therefore, these findings suggest that the fMRI procedure detected drug effects on brain function that were below the threshold that would result in detectable overt changes in behavior. These results, however, must be interpreted with some caution since we did not correct for the multiple statistical comparisons made (multiple ROIs). However, the fact that the brain areas activated by the tasks, and those increased by amphetamine, correspond with areas known to be involved in auditory or motor performance lessens the possibility that the results represent chance findings because of multiple statistical comparisons.

In previous studies examining the effects of stimulants on fMRI activation, Howard et al. (1996) and Gollub et al. (1998) found that stimulants either decreased or had no effect on sensory-induced activation in normal volunteers. Interestingly, amphetamine had the opposite effect in the present study; it increased taskinduced activation during both the auditory-attention and motor tasks and did not decrease activation in any region for either task. It is possible that the previous studies used stimuli with a stronger sensory component, and that amphetamine has a differential effect on sensory- versus task-related changes in fMRI activation. Consistent with this idea, a similar discrepancy is evident between PET studies that have examined the effect of amphetamine on sensory- and task-induced changes in regional cerebral blood flow (Mathew and Wilson 1985; Kahn et al. 1989; Mattay et al. 1996). Nevertheless, the different results in the present study may be related to other methodological differences. For instance, Gollub et al. (1998) used intravenous cocaine rather than oral amphetamine, and Howard et al. (1996) administered a lower dose of oral amphetamine.

In the motor task, we did not measure the subjects' rate of finger tapping, and it is possible that the increase in the activated voxels after amphetamine resulted from an increase in the rate of tapping. This possibility cannot be ruled out, and limits the conclusions that can be drawn from the study. Nevertheless, there are reasons to believe that the increase in the number of active voxels was not simply a result of increased motor activity. The normal, healthy volunteers in the present study were instructed to tap as fast as they could, which typically results in a rate of more than $2 \mathrm{~Hz}$ (Rao et al. 1993). Although Rao et al. (1996) reported that the number of activated voxels increased between tapping rates of 1 and $2 \mathrm{~Hz}$, further increases in tapping rate, in the range of $2-5 \mathrm{~Hz}$, did not affect the number of active voxels. At these higher frequencies, Rao et al. (1996) found that higher rates of tapping were associated with higher percent signal change in the contralateral primary motor cortex. However, in the present study no difference in percent signal change was observed after amphetamine, suggesting that the subjects' rate of tapping remained relatively constant. Thus, although the issue of 


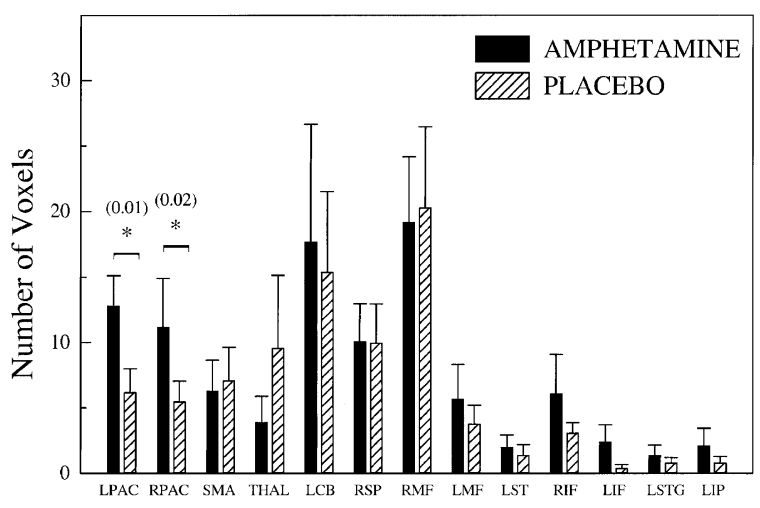

Figure 4. Histogram showing the average number of activated voxels $(4 \times 4 \times 4 \mathrm{~mm})$ in each $\mathrm{ROI}$ during the TD task in the presence of amphetamine or placebo: left primary auditory cortex (LPAC), right primary auditory cortex (RPAC), supplemental (left and right) motor cortex (SMA), thalamus (THAL), left cerebellum (LCB), right superior parietal area (RSP), right middle frontal area (RMF), left middle frontal area (LMF), left superior temporal area (LST), right inferior frontal cortex (RIF), left inferior frontal cortex (LIF), left superior temporal gyrus (LSTG), and left inferior parietal area (LIP). Solid bars indicate the number of active voxels after ingestion of amphetamine, and hashed bars show the number of activated voxels after ingestion of placebo. Data represent the mean \pm SEM. ROIs that are significantly different (LPAC: $p<.01$, RPAC: $p<.02$ ) according to a paired Wilcoxon signed rank test (uncorrected for multiple ROI comparisons) are indicated by $a^{*}$, with the level of significance in parentheses.

whether amphetamine produced overt behavioral effects must be resolved in a future study, the Rao et al. data suggest that the increased number of activated voxels in our study were not due to facilitation of the behavior.

Reaction Time

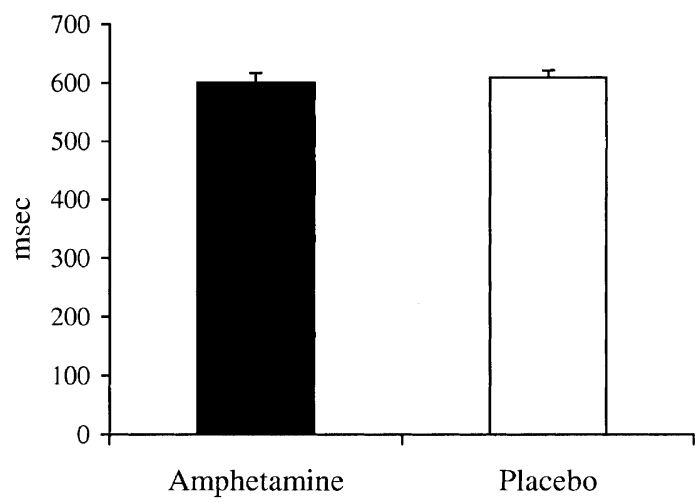

The observed increase in the number of activated voxels during the tasks could be explained by at least two different effects of amphetamine on neuronal activity. First, the drug might have increased the number of neurons recruited to perform the task. This is consistent with the observation in rats that amphetamine increases multiunit neuronal activity (Rebec and Segal 1978; Hansen and McKenzie 1979; Warenycia and McKenzie 1989). It is notable that in our study amphetamine did not produce an fMRI signal in any new brain areas that were not also activated in the placebo condition. This suggests that the recruited neurons would most likely be within the same neural circuit. The second explanation for the observed effect is that the same neurons that are normally activated by the tasks may have been activated to a greater extent in the presence of amphetamine. This is consistent with the observations in laboratory animals that both amphetamine and cocaine appear to increase the gain, or signal-to-noise ratio, by increasing the electrophysiological activity of individual neurons associated with motor function in rodents (Haracz et al. 1993; Rebec et al. 1997; White et al. 1998). It is not possible to separate these two actions from the present results, and the two actions are not mutually exclusive. It is possible that amphetamine may both cause more neurons to be activated and increase the activity of specific neurons associated with the task.

The observed increase in the number of active voxels during the tasks could also be explained by a direct effect of amphetamine on vasculature. For example, amphetamine is known to have vasoconstrictive effects, which can result in alterations of blood flow (Simpson 1976; Angrist et al. 1987; Brauer et al. 1996). Although it seems unlikely, amphetamine may have directly affected blood flow only in the specific brain areas where changes in the activation pattern were observed. That

Response Accuracy

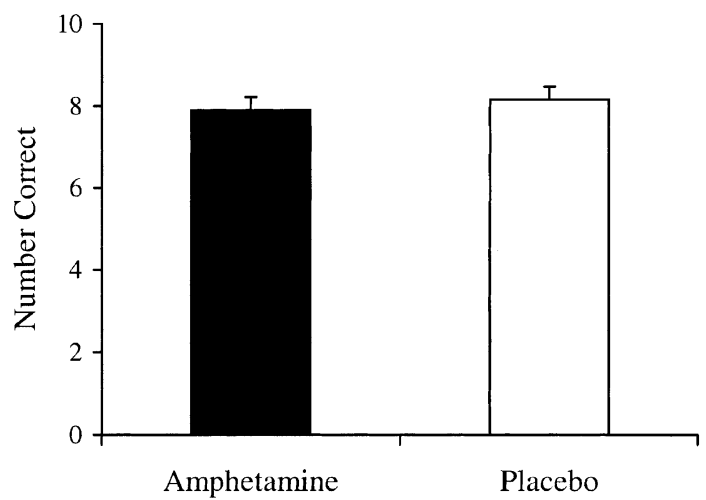

Figure 5. Measures of performance on the Tone Decision task during the fMRI scan. Data represent the mean \pm SEM. 


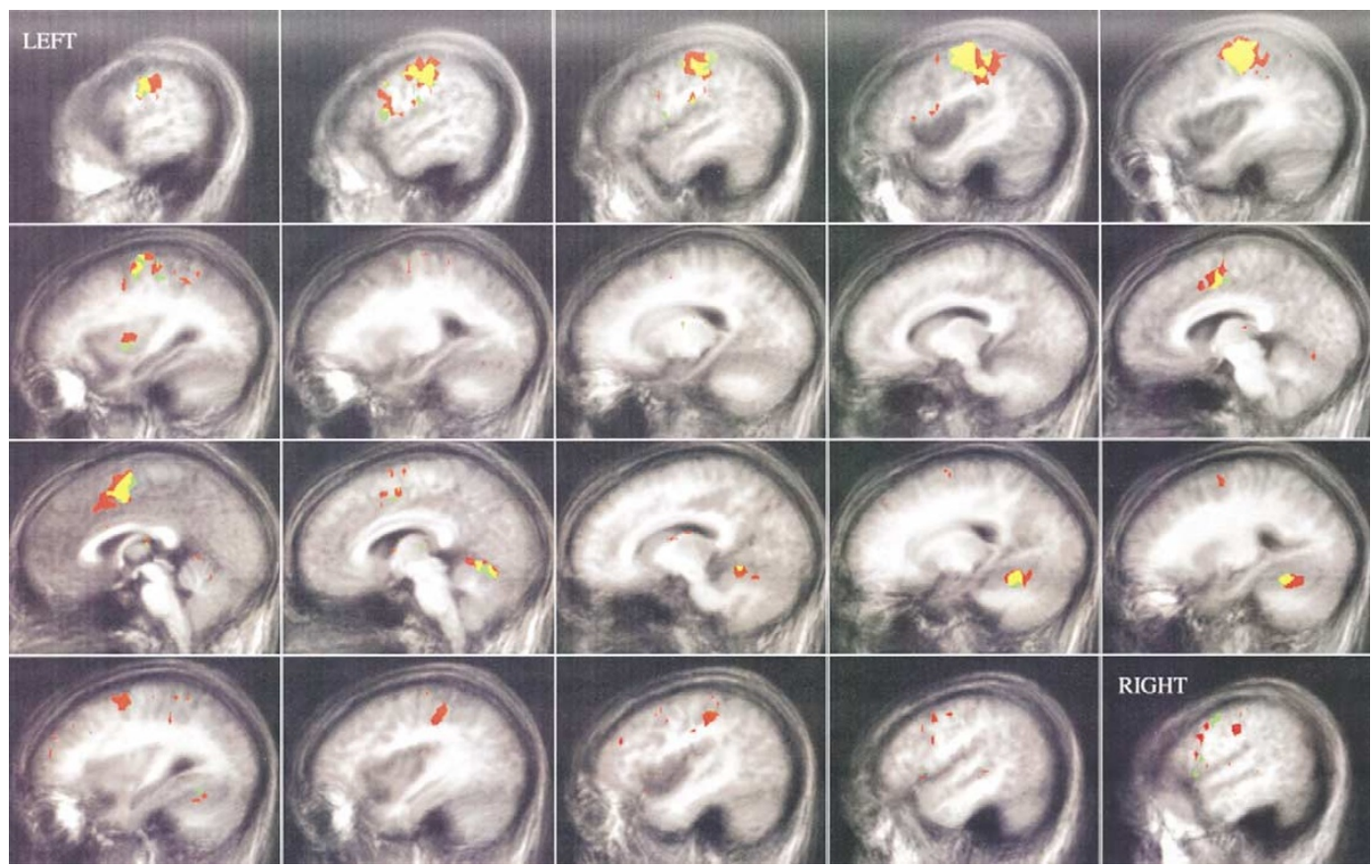

Figure 6. Map of brain activation in the group of subjects ("group-average" activation map) during the right-handed finger-tapping task. Red indicates areas of activation after the subject had ingested amphetamine, and green indicates areas of activation after the subject had ingested placebo. Areas that were active after ingestion of placebo and after ingestion of amphetamine are colored yellow.

is, in those areas, the task may have stimulated a greater increase in blood flow in the presence of amphetamine despite equivalent neuronal activation. It is not possible to resolve this definitively without direct measures of blood flow and a more through understanding of the coupling between neuronal activity and the hemodynamic response. It is notable, however, that in studies that directly measure cerebral blood flow $(\mathrm{CBF})$, there are at least two reports that amphetamine non-significantly decreased cerebral blood flow (Daniel et al. 1991; Kahn et al. 1989), making this explanation less likely.

One limitation of the design and analysis in the current study was that we could only measure the effects of amphetamine on the neuronal activity if it was being modulated by the tasks being performed. Therefore, changes in resting state or baseline neuronal activity could not be measured. One way to use fMRI to measure the effects of amphetamine on the baseline levels of neural activity, independent of task, might be to administer the drug intravenously. Indeed, it has been reported that intravenous infusions of cocaine, another psychostimulant, induced regionally specific fMRI activation (Breiter et al. 1997) that was consistent with known neuroanatomical pathways and anatomical measurements of stimulant effects (Vollenweider et al. 1998).

Many of the affective and cognitive effects of amphetamine are believed to be mediated by dopamine.
However, in the present study, amphetamine increased activation of primary auditory cortex during the TD task and of ipsilateral motor and ipsilateral middle frontal cortices during the FT task areas that are not major projection targets of dopamine pathways. This suggests that these activational effects of amphetamine were secondary to the effects of amphetamine in other brain areas. It is not known how the effects of amphetamine on dopamine function interact with the effects of amphetamine on behaviors not normally associated with dopamine. It is also not known how the effects of dopamine on mood and affect interact with its effects on cognition and motor performance.

Table 4. Average Percent Change of fMRI Signal in Each ROI for FT Task ( \pm SEM)

\begin{tabular}{lcc}
\hline ROI & Amphetamine & Placebo \\
\hline CPSM & $3.3 \pm 0.4$ & $3.3 \pm 0.3$ \\
IPSM & $1.9 \pm 0.5$ & $1.3 \pm 0.5$ \\
SMA & $2.9 \pm 0.4$ & $2.3 \pm 0.5$ \\
ICB & $2.4 \pm 0.4$ & $2.6 \pm 0.4$ \\
CCB & $1.4 \pm 0.7$ & $1.3 \pm 0.5$ \\
RMF & $2.8 \pm 0.6$ & $1.4 \pm 0.5$ \\
LMF & $1.7 \pm 0.6$ & $2.3 \pm 0.7$ \\
RIF & $1.8 \pm 0.8$ & $0.6 \pm 0.6$ \\
LIF & $2.4 \pm 0.7$ & $1.8 \pm 0.6$ \\
THAL & $1.6 \pm 0.7$ & $2.0 \pm 0.9$ \\
PUT & $0.6 \pm 0.4$ & $0.6 \pm 0.3$ \\
\hline
\end{tabular}




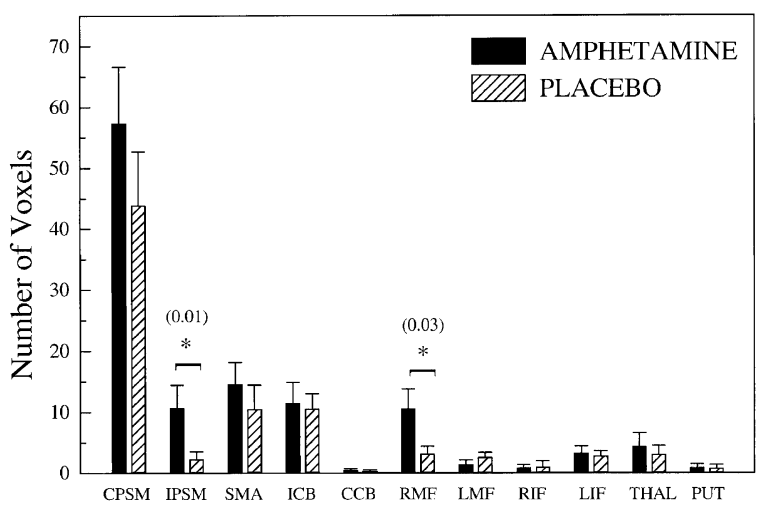

Figure 7. Histogram showing the average number of activated voxels $(4 \times 4 \times 4 \mathrm{~mm})$ in each ROI during the FT task in the presence of amphetamine or placebo: contralateral (left) primary sensorimotor cortex (CPSM), ipsilateral (right) primary sensorimotor cortex (IPSM), supplemental (right and left) motor cortex (SMA), ipsilateral (right) cerebellum (ICB), contralateral (left) cerebellum (CCB), right middle frontal area (RMF), left middle frontal area (LMF), right inferior frontal cortex (RIF), left inferior frontal cortex (LIF), thalamus (THAL), and putamen (PUT). Solid bars indicate the number of active voxels after ingestion of amphetamine, and hashed bars show the number of activated voxels after ingestion of placebo. Error bars indicate the standard error of the mean. ROIs that are significantly different (IPSM: $p<$ .01 , RMF: $p<.03$ ) according to a paired Wilcoxon signed rank test (uncorrected for multiple ROI comparisons) are indicated by $a *$, with the level of significance in parentheses.

In summary, fMRI provided a sensitive measure of regionally specific effects of amphetamine on brain activity related to auditory attention and motor tasks. The goal of future studies will be to determine the functional consequence of the observed increases in activation in primary auditory cortices during a simple tone-decision task and in the ipsilateral primary sensorimotor and right middle frontal cortices during a finger-tapping task. Moreover, it will be important to determine whether the patterns of fMRI activation associated with other cognitive and behavioral tasks are similarly affected by amphetamine.

\section{ACKNOWLEDGMENTS}

The authors would like to thank Alyson Schuster for her help in subject preparation and in running the fMRI experiments. This work was supported in part by grants from the Brain Research Foundation, the Center for Early Childhood Research, General Electric Medical Systems, and the NIH (R01 HD32665, DA02812, 5T32 CA09649).

\section{REFERENCES}

American Psychiatric Association (1994): Diagnostic and
Statistical Manual of Mental Disorders, 4th ed. Washington DC, American Psychiatric Association

Anderson TW (1984): An Introduction to Multivariate Statistical Analysis, 2nd ed. New York, John Wiley and Sons, p 122

Angrist B, Corwin J, Bartlik B, Cooper T (1987): Early pharmacokinetics and clinical effects of oral d-amphetamine in normal subjects. Biol Psychiatry 22:1357-1368

Ardekani BA, Kanno I (1998): Statistical methods for detecting activated regions in functional MRI of the brain. Magn Reson Imag 16:1217-1225

Binder JR, Frost JA, Hammeke TA, Cox RW, Rao SM, Prieto T (1997): Human brain language areas identified by functional magnetic resonance imaging. J Neurosci 17:353-362

Brauer LH, Amber J, de Wit H (1996): Acute tolerance to the subjective but not cardiovascular effects of d-amphetamine in normal, healthy men. J Clin Psychopharmacol 16:72-76

Brauer LH, de Wit H (1995): Role of dopamine in d-amphetamine-induced euphoria in normal, healthy volunteers. Exp Clin Psychopharmacol 3:371-381

Brauer LH, de Wit H (1997): High dose pimozide does not block amphetamine-induced euphoria in normal volunteers. Pharmacol Biochem Behav 56:265-272

Breiter HC, Gollub RL, Weisskoff RM, Kennedy DN, Makris N, Berke JD, Goodman JM, Kantor HL, Gastfriend DR, Riorden JP, Mathew RT, Rosen BR, Hyman SE (1997): Acute effects of cocaine on human brain activity and emotion. Neuron 19:591-611

Bye C, Munro-Faure AD, Peck AW, Young PA (1973): A comparison of the effects of 1-benzylpiperazine and dexamphetamine on human performance tests. Eur J Clin Pharmacol 6:163-169

Cao Y, Towle VL, Levin DN, Balter JM (1993): Functional mapping of human motor cortical activation with conventional MR imaging at 1.5 T. J Magn Reson Imag 3:869-875

Cohen MS, Bookheimer SY (1994): Localization of brain function using magnetic resonance imaging. Trends Neurosci 17:268-277

Cox RW (1996): AFNI: Software for analysis and visualization of functional magnetic resonance neuroimages. Comp and Biomed Res 29:162-173

Daniel DG, Weinberger DR, Jones DW, Zingun JR, Coppola R, Handel S, Bigelow LB, Goldberg TE, Berman KF, Kleinman JE (1991): The effect of amphetamine on regional cerebral blood flow during cognitive activation in schizophrenia. J Neurosci 11:1907-1917

de Wit H, Clark M, Brauer LH (1997): Effects of d-amphetamine in grouped versus isolated humans. Pharmacol Biochem Behav 57:333-340

Fink GR, Frackowiak RSJ, Pietrzyk U, Passingham RE (1997): Multiple nonprimary motor areas in the human cortex. J Neuorophysiol 77: 2164-2174

Fischman MW, Foltin RW (1991): Utility of subjective-effects measurements in assessing abuse liability of drugs in humans. Br J Addict 86:1563-1570

Folstein MF, Luria R (1973): Reliability, validity, and clinical application of the Visual Analogue Mood Scale. Psychol Med 3:479-486 
Foltin RW, Fischman MW (1991a): Assessment of abuse liability of stimulant drugs in humans: A methodological survey. Drug Alcohol Depend 28:3-48

Foltin RW, Fischman MW (1991b): Methods for the assessment of abuse liability of psychomotor stimulants and anorectic agents in humans. Br J Addict 86:1633-1640

Forman SD, Cohen JD, Fitzgerald M, Eddy WF, Mintun MA, Noll DC (1995): Improved assessment of significant activation in functional magnetic resonance imaging (fMRI): Use of a cluster-size threshold. Magn Reson Med 33:636-647

Gollub RL, Breiter HC, Kantor H, Kennedy D, Gastfriend D, Mathew RT, Makris N, Guimaraes A, Riorden J, Campbell T, Foley M, Hyman SE, Rosen B, Weisskoff R (1998): Cocaine decreases cortical cerebral blood flow but does not obscure regional activation in functional magnetic resonance imaging in human subjects. J Cereb Blood Flow Metab 18:724-734

Hamilton MJ, Smith PR, Peck AW (1983): Effects of bupropion, nomifensine and dexamphetamine on performance, subjective feelings, autonomic variables and electroencephalogram in healthy volunteers. Br J Clin Pharmacol 15:367-374

Hansen EL, McKenzie GM (1979): Dexamphetamine increases striatal neuronal firing in freely moving rats. Neuropharmacology 18:547-552

Haracz JL, Tschanz JT, Wang Z, White IM, Rebec GV (1993): Striatal single-unit responses to amphetamine and neuroleptics in freely moving rats. Neurosci Biobehav Rev 17:1-12

Howard RJ, Ellis C, Bullmore ET, Brammer M, Mellers JD, Woodruff PW, David AS, Simmons A, Williams SC, Parkes JD (1996): Functional echoplanar brain imaging correlates of amphetamine administration to normal subjects and subjects with the narcoleptic syndrome. Magn Reson Imag 14:1013-1016

Kahn DA, Prohovnik I, Lucas LR, Sackeim HA (1989): Dissociated effects of amphetamine on arousal and cortical blood flow in humans. Biol Psychiatry 25:755-767

Kleinschmidt A, Bruhn H, Kruger G, Merboldt K-D, Stoppe G, Frahm J (1999): Effects of sedation, stimulation, and placebo on cerebral blood oxygenation: A magnetic resonance neuroimaging study of psychotropic drug action. NMR Biomed 12:286-292

Koelega HS (1993): Stimulant drugs and vigilance performance: A review. Psychopharmacology 111:1-16

Maas LC, Lukas SE, Kaufman MJ, Weiss RD, Daniels SL, Rogers VW, Kukes TJ, Renshaw PF (1998): Functional MRI of human brain activation during cue-induced cocaine craving. Am J Psychiatry 1:124-126

Martin WR, Sloan JW, Sapira JD, Jasinski DR (1971): Physiologic, subjective, and behavioral effects of amphetamine, methamphetamine, ephedrine, phenmetrazine, and methylphenidate in man. Clin Pharmacol Ther 12:245-258
Mathew RJ, Wilson WH (1985): Dextroamphetamine-induced changes in regional cerebral blood flow. Psychopharmacology 87:298-302

Mattay VS, Berman KF, Ostrem JL, Esposito G, Van Horn JD, Bigelow LB, Weinberger DR (1996): Dextroamphetamine enhances "neural network-specific" physiological signals: A positron-emission tomography rCBF study. J Neurosci 16:4816-4822

Olfield RC (1971): The assessment and analysis of handedness: The Edinburgh Inventory. Neuropsychologia 9: 97-113

Rao SM, Bandettini PA, Binder JR, Bobholz JA, Hammeke TA, Stein EA, Hyde JS (1996): Relationship between finger movement rate and functional magnetic resonance signal change in human primary motor cortex. J Cereb Blood Flow Metab 16:1250-1254

Rao SM, Binder JR, Bandettini PA, Hammeke TA, Yetkin FZ, Jesmanowicz A, Lisk LM, Morris GL, Mueller WM, Estkowski LD, Wong EC, Haughton VM, Hyde JS (1993): Functional magnetic resonance imaging of complex human movements. Neurology 43:2311-2318

Rebec GV, Segal DS (1978): Dose-dependent biphasic alterations in the spontaneous activity of neurons in the rat neostriatum produced by d-amphetamine and methylphenidate. Brain Res 150:353-366

Rebec GV, White IM, Puotz JK (1997): Responses of neurons in dorsal striatum during amphetamine-induced focused stereotypy. Psychopharmacology 130:343-351

Schubert T, von Cramon DY, Niendorf T, Pollmann S, Bublak P (1998): Cortical areas and the control of selfdetermined finger movements: An fMRI study. Neuroreport 9:3171-3176

Simpson LL (1976): The effect of behavioral stimulant doses of amphetamine on blood pressure. Arch Gen Psychiatry 33:691-695

Talairach J, Tournoux P (1998): Co-Planar Sterotaxic Atlas of the Human Brain. New York, Thieme Med Publications

Vollenweider FX, Maguire RP, Leenders KL, Mathys K, Angst J (1998): Effects of high amphetamine dose on mood and cerebral glucose metabolism in normal volunteers using positron emission tomography (PET). Psychiatry Res 83:149-162

Wachtel SR, de Wit H (1999): Subjective and behavioral effects of repeated d-amphetamine in humans. Behav Pharmacol 10:271-281

Warenycia MW, McKenzie GM (1989): Pharmacological activity profiles of dopamine D-1 and D-2 receptor agonist and antagonists on striatal neuronal activity and the response to dexamphetamine in freely moving rats. Gen Pharmacol 20:295-301

White IM, Doubles L, Rebec GV (1998): Cocaine-induced activation of striatal neurons during focused stereotypy in rats. Brain Res 810:146-152

Woods RP, Cherry SR, Mazziotta JC (1992): Rapid automated algorithm for aligning and reslicing PET images. J Comput Assist Tomogr 16:620-633 